\title{
Neurosteroid allopregnanolone (3a,5a-THP) inhibits inflammatory signals induced by activated MyD88-dependent toll-like receptors
}

\author{
Irina Balan', Laure Aurelian², Riana Schleicher', Giorgia Boero ${ }^{1}$, Todd O'Buckley (1) and A. Leslie Morrow (1)
}

\begin{abstract}
We have shown that endogenous neurosteroids, including pregnenolone and 3a,5a-THP inhibit toll-like receptor 4 (TLR4) signal activation in mouse macrophages and the brain of alcohol-preferring $(P)$ rat, which exhibits innate TLR4 signal activation. The current studies were designed to examine whether other activated TLR signals are similarly inhibited by 3a,5a-THP. We report that 3a,5a-THP inhibits selective agonist-mediated activation of TLR2 and TLR7, but not TLR3 signaling in the RAW246.7 macrophage cell line. The TLR4 and TLR7 signals are innately activated in the amygdala and NAc from P rat brains and inhibited by $3 a, 5 a-T H P$. The TLR2 and TLR3 signals are not activated in P rat brain and they are not affected by 3a,5a-THP. Co-immunoprecipitation studies indicate that 3a,5a-THP inhibits the binding of MyD88 with TLR4 or TLR7 in P rat brain, but the levels of TLR4 co-precipitating with TRIF are not altered by 3a,5a-THP treatment. Collectively, the data indicate that 3a,5a-THP inhibits MyD88- but not TRIF-dependent TLR signal activation and the production of pro-inflammatory mediators through its ability to block TLR-MyD88 binding. These results have applicability to many conditions involving pro-inflammatory TLR activation of cytokines, chemokines, and interferons and support the use of 3a,5a-THP as a therapeutic for inflammatory disease.
\end{abstract}

\section{Introduction}

Toll-like receptors (TLRs) are a family of pattern recognition receptors that are expressed in macrophages, microglia, astrocytes, and neurons and contribute to proinflammatory, innate, and neuroimmune responses. They are located on the cell surface (TLR1, 2, 4, 5, 6, 11, 12) or endosomes (TLR3, 7, 8, 9, 13) and recognize molecular signatures that initiate signaling pathways for cytokine and chemokine expression, which depend on the binding of the adaptor proteins myeloid differentiation primary response 88 (MyD88) or TIR-domain-containing adapterinducing interferon- $\beta$ (TRIF) ${ }^{1-6}$. Most TLRs (except TLR3 and 4) signal exclusively through MyD88-dependent

Correspondence: A. Leslie Morrow (morrow@med.unc.edu)

'Department of Psychiatry, Department of Pharmacology, Bowles Center for Alcohol Studies, University of North Carolina at Chapel Hill, School of Medicine, Chapel Hill, NC 27599, USA

${ }^{2}$ Stanford University School of Medicine, Stanford, CA 94305, USA

These authors contributed equally: Irina Balan, Laure Aurelian pathways to produce pro-inflammatory cytokines and chemokines or activate interferon pathways. TLR3 signals exclusively through TRIF-dependent pathways and TLR4 signals through both MyD88- and TRIF-dependent pathways. TLR signal activation and excessive cytokine production are implicated in the etiology of depression ${ }^{7,8}$, alcohol use disorders and other addictions ${ }^{9,10}$, traumatic brain injury ${ }^{11}$, neurodegeneration ${ }^{3,12,13}$, ischemia ${ }^{14}$, epilepsy ${ }^{15,16}$, and various systemic inflammatory conditions, including sepsis and Covid-19 17,18 .

Neurosteroid allopregnanolone $\quad((3 \alpha, 5 \alpha) 3$-hydroxypregnan-20-one, $3 \alpha, 5 \alpha-\mathrm{THP})$ is synthesized in adrenals, gonads, and brain from cholesterol or sterol precursors. It acts upon synaptic and extrasynaptic $\gamma$-aminobutyric acid type $\mathrm{A}\left(\mathrm{GABA}_{\mathrm{A}}\right)$ receptors to mediate phasic and tonic inhibition $^{19,20}$, has anesthetic, anticonvulsant, sedative, and anxiolytic effects ${ }^{21}$, and modulates the hypothalamic pituitary adrenal axis to reduce stress activation ${ }^{22}$. Significantly, $3 \alpha, 5 \alpha$-THP and/or its precursors progesterone

\section{(c) The Author(s) 2021}

(c) (i) Open Access This article is licensed under a Creative Commons Attribution 4.0 International License, which permits use, sharing, adaptation, distribution and reproduction c. in any medium or format, as long as you give appropriate credit to the original author(s) and the source, provide a link to the Creative Commons license, and indicate if changes were made. The images or other third party material in this article are included in the article's Creative Commons license, unless indicated otherwise in a credit line to the material. If material is not included in the article's Creative Commons license and your intended use is not permitted by statutory regulation or exceeds the permitted use, you will need to obtain permission directly from the copyright holder. To view a copy of this license, visit http://creativecommons.org/licenses/by/4.0/. 
and pregnenolone, were shown to be effective in clinical studies of traumatic brain injury ${ }^{23}$, schizophrenia ${ }^{24}$, cocaine craving $^{25}$, and post-partum depression ${ }^{26}$, identifying them as promising therapeutics ${ }^{27,28}$. Remarkably, these diverse conditions all exhibit pro-inflammatory immune and neuroimmune activation.

Recent studies showed that $3 \alpha, 5 \alpha$-THP, progesterone, and pregnenolone inhibit TLR4 signaling and its cognate pro-inflammatory factors, including monocyte chemoattractant protein-1 (MCP-1), high mobility group box 1 (HMGB1), and tumor necrosis factor alpha (TNF- $\alpha$ ) in RAW264.7 cells and brain ${ }^{29-31}$. We showed that $3 \alpha, 5 \alpha-$ THP inhibits the TLR activation mechanisms involving TLR4 binding to myeloid differentiation factor 2 (MD2), MyD88, and $\mathrm{GABA}_{\mathrm{A}} \alpha 2$ subunits $^{31}$. Here, we report that $3 \alpha, 5 \alpha$-THP inhibits TLR activation via MyD88- but not TRIF-dependent signals to regulate a broad spectrum of pro-inflammatory responses in the innate immune system and the brain.

\section{Materials and methods Cells and reagents}

Mouse macrophage/monocyte RAW264.7 cells were obtained from American Type Culture Collection (Manassas, VA, USA) and grown as previously described ${ }^{31}$ and details are provided in Supplemental Information (SI) Materials and Methods. The selective agonist for TLR2 [Pam ${ }_{3}$ Cys-Ser-(Lys) ${ }_{4}$ hydrochloride (Pam3Cys; $10 \mu \mathrm{g} / \mathrm{ml}$ )] (Cat. \#506350, Sigma-Aldrich, Saint Louis, MO, USA) was added to the cultures alone, or together with $3 \alpha, 5 \alpha$-THP $(1.0 \mu \mathrm{M})$ in DMEM (without FBS and antibiotics) for $30 \mathrm{~min}$ and cells were harvested after $24 \mathrm{~h}$. Selective agonists for TLR3 [polyinosinic-polycytidylic acid potassium salt (Poly(I:C); $25 \mu \mathrm{g} / \mathrm{ml})$ ] (Cat. \#P9582, SigmaAldrich) or TLR7 [imiquimod (IMQ); $3 \mu \mathrm{g} / \mathrm{ml}$ ] (Cat. \#tlrlimqs, InvivoGen, San Diego, CA, USA) were added to the cultures alone, or together with $3 \alpha, 5 \alpha-\mathrm{THP}(1.0 \mu \mathrm{M})$ in DMEM (without FBS and antibiotics) $24 \mathrm{~h}$ before cell collection.

\section{Antibodies}

Antibodies were commercially obtained, validated and used according to the manufacturer's instructions. Primary antibodies, their host species, clonality and dilution are listed in Table S1. Horseradish peroxidase-labeled secondary antibodies were anti-rabbit (Cat. \# 7074, RRID: AB_2099233, Cell Signaling Technology), anti-mouse (Cat\# 7076, RRID: AB_330924, Cell Signaling Technology), and anti-goat IgG (Cat\# A24452, RRID: AB_2535921, Thermo Fisher Scientific, Waltham, MA, USA).

\section{Animals}

Male and female alcohol-preferring (P) (males: $N=38$; females: $N=38$ ) and non-preferring (NP) rats (males:
$N=6$; females: $N=6)(3-4$ months old; $250-550$ g) were obtained from the Alcohol Research Center, Indiana University School of Medicine, double housed in Plexiglas cages, given food and water ad libitum and maintained on a $12 \mathrm{~h}$ light-dark cycle (light onset at $0700 \mathrm{~h}$ ). We examined $3 \alpha, 5 \alpha-$ THP regulation of TLR signaling in alcohol-P rats because the selective breeding of these rats for alcohol preference resulted in innate activation of TLR4 and nuclear factor kappa-light-chain-enhancer of activated $B$ cells (NF-kB) signals ${ }^{9,32-36}$ as well as numerous pathological behavioral properties, including anxiety-like behavior $^{37,38}$, impulsivity ${ }^{33}$, and stress reactivity ${ }^{39}$. Procedures followed NIH Guidelines under Institutional Animal Care and Use Committee approved protocols at the University of North Carolina, School of Medicine. All studies were conducted in the morning to avoid potential circadian fluctuations in neurosteroids ${ }^{40,41}$. P rats were randomly given $3 \alpha, 5 \alpha-$ THP $(15 \mathrm{mg} / \mathrm{kg})$ (males: $N=16$; females: $N=16) \quad$ or $\quad$ vehicle $\quad(45 \% \quad \mathrm{w} / \mathrm{v} \quad$ 2-hydroxypropyl$\beta$-cyclodextrin) (males: $N=16$; females: $N=16$ ) by intraperitoneal (IP) injection and sacrificed $30 \mathrm{~min}$ later. Collected brains were stored at $-80^{\circ} \mathrm{C}$ and nucleus accumbens (NAc) and amygdala were dissected on ice using a brain matrix. This dose and time point were selected based on previous studies showing that $3 \alpha, 5 \alpha-$ THP has anxiolytic ${ }^{42}$ and anticonvulsant ${ }^{43}$, but not hypnotic effects ${ }^{44}$, and we have previously demonstrated that it inhibits TLR4 pathway activation ${ }^{31}$.

\section{Immunoblotting and co-immunoprecipitation}

Protein extraction and assay were as previously described $^{31,32}$ and details are provided in SI, Materials and Methods. Results are expressed as mean $\beta$-actin-adjusted densitometric units \pm SEM or mean MyD88- or TRIFadjusted densitometric units \pm SEM.

\section{ELISA}

Protein extraction was identical to that for immunoblotting and detailed in SI, Materials and Methods. Protein extracts were assayed with ELISA kits (Raybiotech, Norcross, GA, USA) for MCP-1 (Cat. \#ERC-MCP-1-CL), TNF- $\alpha$ (Cat. \#ELM-TNFa-CL-1), or pIRF3 (Cat. \#PELIRF3-S386) as per the manufacturer's instructions. Results are expressed as picograms/milligram total protein $(\mathrm{pg} / \mathrm{mg})$ or relative units/milligram total protein (RU/mg).

\section{Statistics}

RAW264.7 cells treated with TLR agonists with/without $3 \alpha, 5 \alpha-$ THP ( $n=5-10 /$ grp) were analyzed using one-way analysis of variance (ANOVA) followed by Tukey's post hoc test. Effects of $3 \alpha, 5 \alpha-$ THP on RAW264.7 cells $(n=6 /$ grp) that were not treated with TLR agonists, were analyzed by $t$-test. Four separate cohorts of animals were used. Brain tissues $(n=8 /$ grp $)$ from male and female 
$\mathrm{P}$ rats that were given $3 \alpha, 5 \alpha$-THP or vehicle (Cohort 1 ) were analyzed using two-way ANOVA followed by Tukey's post hoc test. Brain tissues $(n=8 /$ grp $)$ from female $\mathrm{P}$ rats that were given $3 \alpha, 5 \alpha$-THP or vehicle (Cohort 2), co-immunoprecipitation $\quad(n=3-4 /$ grp $)$ (Cohort 2 and 3) and comparison of naïve untreated NP ( $n=6 /$ grp) vs. P ( $n=6 /$ grp) rat brain tissues (Cohort 4 ), were analyzed by $t$-test using Graphpad Prism 8.3.1. $P<0.05$ was considered statistically significant.

\section{Results}

3a,5a-THP inhibits the activation of MyD88-dependent TLR pathways in RAW264.7 cells

We have previously shown that $3 \alpha, 5 \alpha$-THP inhibits the activated TLR4 signaling pathway in cultured cells, but it has no effect on the steady state non-activated pathway ${ }^{31}$. Inhibition is independent of the cell type and the activating agonist. It was seen in both lipopolysaccharide (LPS)-treated RAW246.7 macrophage/monocyte cells and neuronal $\mathrm{N} 2 \mathrm{a}$ cells, where the activator is the $\mathrm{GABA}_{\mathrm{A}}$ receptor $\alpha 2$ subunit $^{31,45}$. Inhibition levels were similar for $3 \alpha, 5 \alpha-\mathrm{THP}$ at 0.5 and $1.0 \mu \mathrm{M}^{31}$. Pathway inhibition included blocking of the phosphorylation (activation) of canonical signaling members including transforming growth factor beta-activated kinase 1 (TAK1), NF-kB p65, and cAMP-response element-binding protein (CREB) and the resulting expression of $\mathrm{MCP}-1^{31}$.

To examine whether $3 \alpha, 5 \alpha-$ THP also inhibits activation of other TLR signals and pathway members, we first studied its effect in RAW246.7 cells, an established cell line model of adaptive and innate immunity ${ }^{46,47}$, using selective TLR2, 3 and 7 ligands. We focused on TLRs located on the plasma membrane (TLR2) or endosomes (TLR7, TLR3) that signal exclusively through MyD88 (TLR2, TLR7) or TRIF (TLR3) binding ${ }^{2,48-52}$. Cells were treated with the TLR2 agonist Pam3Cys $(10 \mu \mathrm{g} / \mathrm{ml}$, $30 \mathrm{~min}$ and cells harvested after $24 \mathrm{~h}$ ), the TLR7 agonist IMQ $(3 \mu \mathrm{g} / \mathrm{ml} ; 24 \mathrm{~h})$ or the TLR3 agonist Poly(I:C) $(25 \mu \mathrm{g} / \mathrm{ml} ; 24 \mathrm{~h})$, in the absence or presence of $3 \alpha, 5 \alpha-$ THP $(1.0 \mu \mathrm{M})$. Cell extracts were assayed for canonical members of activated pathways, including tumor necrosis factor receptor-associated factor 6 (TRAF6), activated (phosphorylated) extracellular signal-regulated kinase $1 / 2$ (pERK1/2), pCREB, activating transcription factor 2 (pATF2), the TLR7-associated activated (phosphorylated) transcription factor interferon regulatory factor 7 (pIRF7), the TLR3-associated activated transcription factor IRF3 (pIRF3) and the cytokines/chemokines TNF$\alpha$ and interferon gamma-induced protein 10 (IP-10; also known as CXCL10) $)^{1,53-55}$. The ligands and $3 \alpha, 5 \alpha-\mathrm{THP}$ concentrations were selected based on previous findings of maximal effects ${ }^{31,56-58}$. The effect of $3 \alpha, 5 \alpha$-THP on cells that were not treated with the TLR agonists was studied in parallel.
Pam3Cys caused a significant increase in the levels of TRAF6 (66.3 $\pm 9.7 \%$; one-way ANOVA, Tukey's post hoc test: $p=0.0158, n=8 /$ grp$)$, pERK $1 / 2(44.6 \pm 11.9 \%$; oneway ANOVA, Tukey's post hoc test: $p=0.0191, n=10 /$ grp), pCREB ( $48.2 \pm 12.2 \%$; one-way ANOVA, Tukey's post hoc test: $p=0.0426, n=10 /$ grp$),$ pATF2 $(54.4 \pm$ 10.1\%; one-way ANOVA, Tukey's post hoc test: $p=$ $0.0443, n=10 /$ grp $)$ and TNF- $\alpha(84.8 \pm 9.3 \%$; one-way ANOVA, Tukey's post hoc test: $p=0.0250, n=5$ /grp) relative to untreated (CTL) cells. $3 \alpha, 5 \alpha$-THP inhibited the Pam3Cys effect on TRAF6 by $56.5 \pm 8.7 \%$ (One-way ANOVA: $F(2,21)=5.453, p=0.0124, n=8 /$ grp $)$, pERK1/ 2 by $55.3 \pm 3.7 \%$ (One-way ANOVA: $F(2,27)=7.314, p=$ $0.0029, n=10 /$ grp), pCREB by $63.8 \pm 3.1 \%$ (One-way ANOVA: $F(2,27)=6.207, p=0.0061, n=10 /$ grp $)$, pATF2 by $60.9 \pm 5.1 \%$ (One-way ANOVA: $F(2,27)=4.854, p=$ $0.0158, n=10 /$ grp) and TNF- $\alpha$ by $76.2 \pm 4.7 \%$ (One-way ANOVA: $F(2,12)=5.667, p=0.0185, n=5 /$ grp) (Fig. 1a). The TLR7 agonist IMQ increased the levels of PIRF7 by $33.4 \pm 4.6 \%$ (One-way ANOVA, Tukey's post hoc test: $p=0.0006, n=9 /$ grp) and TNF- $\alpha$ by $49.3 \pm 9.2 \%$ (Oneway ANOVA, Tukey's post hoc test: $p=0.0103, n=5$ / grp) relative to CTL cells. $3 \alpha, 5 \alpha$-THP inhibited the increase in pIRF7 by $32.6 \pm 6.0 \%$ (One-way ANOVA: $F(2,24)=12.40, p=0.0002, n=9 /$ grp $)$ and the increase in TNF- $\alpha$ by $55.0 \pm 5.1 \%$ (One-way ANOVA: $F(2,12)=$ 9.520, $p=0.0033, n=5$ /grp), respectively (Fig. 1b).

The levels of pIRF3, IP-10, and TNF- $\alpha$ were also significantly increased in cells treated with the TLR3 agonist Poly(I:C) [pIRF3: $30.7 \pm 7.3 \%$ (One-way ANOVA, Tukey's post hoc test: $p=0.0391, n=5 /$ grp), IP-10: $97.3 \pm 0.6 \%$ (One-way ANOVA, Tukey's post hoc test: $p=0.0142$, $n=6 /$ grp) and TNF- $\alpha$ : $87.7 \pm 7.3 \%$ (One-way ANOVA, Tukey's post hoc test: $p=0.0471, n=5 /$ grp), respectively], but these effects were not inhibited by $3 \alpha, 5 \alpha$-THP (One-way ANOVA, Tukey's post hoc test: $p=0.6881$, $p=0.8291$, and $p=0.8892$ for pIRF3 ( $n=5 /$ grp), IP- 10 ( $n=6 /$ grp), and TNF- $\alpha$ ( $n=5 /$ grp), respectively) (Fig. 1c). Consistent with our previous findings ${ }^{31}, 3 \alpha, 5 \alpha$-THP did not alter $(t$-test, $p>0.05)$ the expression of any pathway member in cells that were not treated with TLR agonists (Fig. 1d) indicating that $3 \alpha, 5 \alpha$-THP specifically targets only the activated TLR signals. Collectively, the data indicate that $3 \alpha, 5 \alpha-$ THP inhibits the activation of both cell surface and endosomal TLR signals, but only if they are MyD88-dependent.

\section{3a,5a-THP inhibits the innately activated TLR4 and TLR7 signals in the $P$ rat brain}

Neuroimmune signaling in the brain is now well established as playing an important role in brain function and it involves cross talk between multiple cell types including neurons, glia, and endothelial cells ${ }^{10,59-61}$. We have previously shown that $3 \alpha, 5 \alpha$-THP inhibits the 


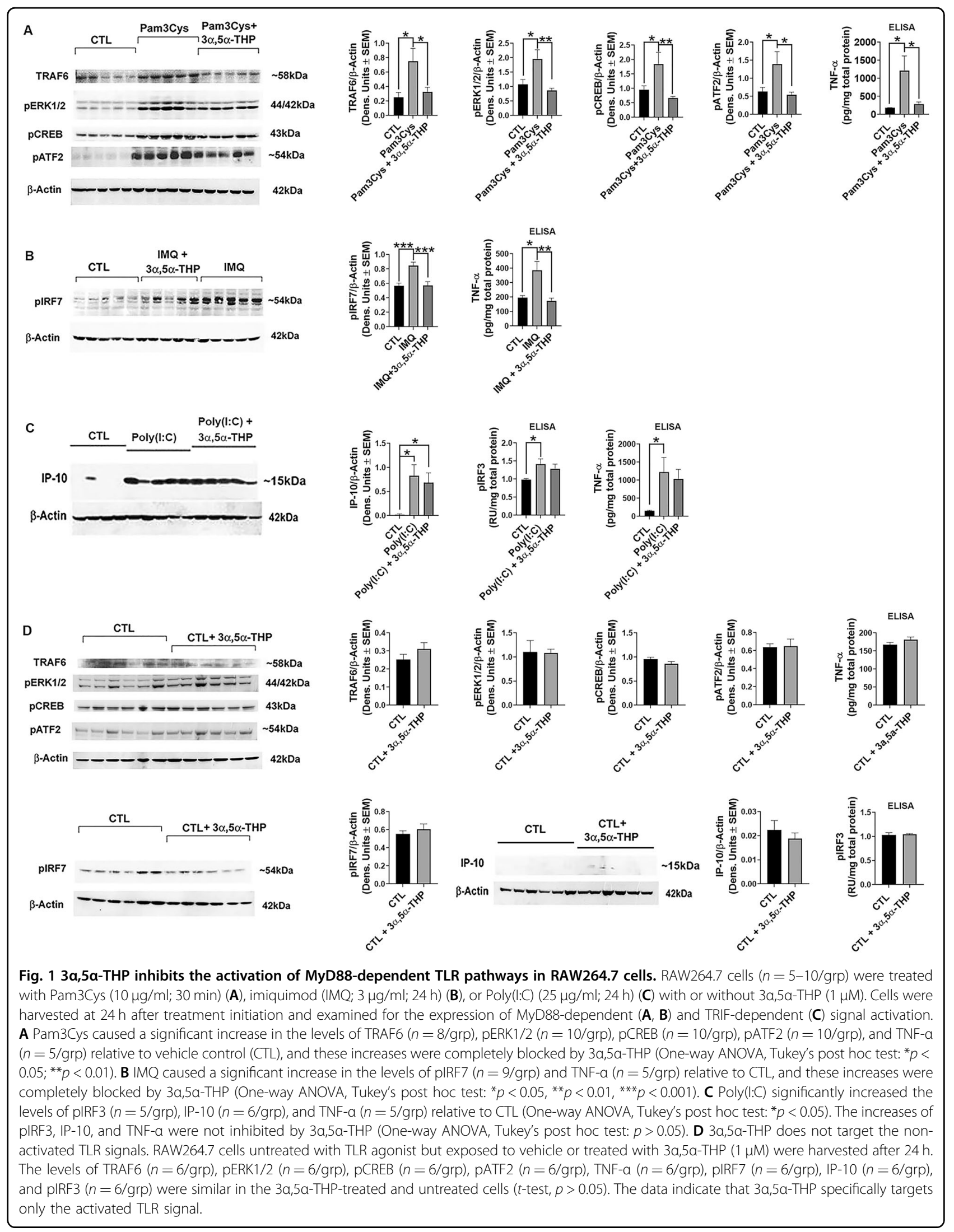


innately activated TLR4 signal in the brain of male alcohol-P rats (ventral tegmental area) ${ }^{31}$. However, its effect on TLR4 signaling in female P rats, the potential existence of other innately activated TLR signaling pathways in male and female $P$ rats, and their inhibition by $3 \alpha, 5 \alpha-$ THP, remained unclear.

Two series of experiments were done in order to address these questions. In a first series of experiments we examined the effect of $3 \alpha, 5 \alpha$-THP on the innately activated TLR4 signal in male and female $P$ rats. Male and female $P$ rats $(n=8 /$ grp $)$ were treated $(30 \mathrm{~min})$ with $3 \alpha, 5 \alpha-$ THP $(15 \mathrm{mg} / \mathrm{kg})$ or vehicle control $(45 \% \mathrm{w} / \mathrm{v}$ 2-hydroxypropyl- $\beta$-cyclodextrin) as previously described ${ }^{31}$ and the expression of MCP-1, [indicative of TLR4 activation ${ }^{35,59}$ ] was assessed in the amygdala and NAc. Its effect on TLR4 was studied in parallel. These brain sites were chosen based on our previous findings that the TLR4 signal is innately activated in the $\mathrm{P}$ rats NAc and the central nucleus of the amygdala, where it is involved in binge drinking $^{32,34,35,59} .3 \alpha, 5 \alpha$-THP inhibited MCP-1 expression both in the amygdala (Two-way ANOVA: $F(1,28)=20.92$, $p<0.0001, n=8 /$ grp) and NAc (Two-way ANOVA: $F$ $(1,28)=21.14, p<0.0001, n=8 /$ grp $)$. The reduction of MCP-1 was similar in males $(57.7 \pm 8.2 \%$ by two-way ANOVA, Tukey's post hoc test: $p=0.0049, n=8$ /grp) and females $(47.3 \pm 9.7 \%$ by Two-way ANOVA, Tukey's post hoc test: $p=0.046, n=8$ /grp) in the amygdala. In the NAc, by contrast, inhibition was only $26.2 \pm 6.4 \%$ (Twoway ANOVA, Tukey's post hoc test: $p=0.0077, n=8 /$ grp) in males compared to $41.7 \pm 3.0 \%$ (Two-way ANOVA, Tukey's post hoc test: $p=0.0282, n=8 /$ grp) in females. In the amygdala, the levels of MCP-1 were similar in males and females (Two-way ANOVA: $F(1,28)=0.02030, p=$ $0.8877, n=8$ /grp) but in the NAc, the MCP-1 levels were significantly higher in males than females (Two-way ANOVA: $F(1,28)=72.27, p<0.0001, n=8 /$ grp). Specifically, the total levels of $\mathrm{MCP}-1$ in the NAc were significantly higher in males than females both in the absence of $3 \alpha, 5 \alpha$-THP $(46.7 \pm 4.1 \%$ by Two-way ANOVA, Tukey's post hoc test: $p<0.0001, n=8 /$ grp) and in its presence $(57.9 \pm 5.2 \%$ by Two-way ANOVA, Tukey's post hoc test: $p<0.0001, n=8 /$ grp), identifying the NAc as a brain site with sex differences for TLR4 activation (Fig. 2a). Consistent with our previous findings ${ }^{31}$, the levels of TLR4 were not altered by $3 \alpha, 5 \alpha-$ THP (Two-way ANOVA: $F(1,28)=0.4218, p=0.5213, n=8 /$ grp $)$ and they were similar in male and female NAc tissues (Two-way ANOVA: $F(1,28)=0.06052, p=0.8075, n=8 /$ grp) (Fig. 2b). Collectively, the data indicate that $3 \alpha, 5 \alpha$-THP inhibits TLR4 signal activation in both males and females, but the activation levels are significantly higher in the NAc from males than females, even in the presence of $3 \alpha, 5 \alpha$-THP.

In the second series of experiments, we asked whether TLR signaling pathways other than TLR4 are also innately activated in the NAc from P but not NP rats, and whether these are inhibited by $3 \alpha, 5 \alpha$-THP. NAc tissues from male and female $\mathrm{P}(n=6 / \mathrm{grp})$ and NP $(n=6 /$ grp $)$ rats were assayed for the expression of TLR7 and pIRF7, which is indicative of TLR7 signal activation ${ }^{1,53}$. The levels of TLR7 (30.4 $\pm 7.7 \%$ by $t$-test: $t=2.827, \mathrm{df}=10, p=0.0179$, $n=6 /$ grp $)$ and pIRF7 $(61.8 \pm 18.3 \%$ by $t$-test: $t=2.240$, $\mathrm{df}=10, p=0.0490, n=6 /$ grp) were significantly higher in female $P$ than NP rats, consistent with innate TLR7 activation in female $\mathrm{P}$ rats (Fig. 2c). However, in males, the levels of TLR7 ( $t$-test: $t=0.5318, \mathrm{df}=10, p=0.6065$, $n=6 /$ grp $)$ and pIRF7 ( $t$-test: $t=0.3349, \mathrm{df}=10, p=$ $0.7446, n=6 /$ grp) were the same in $\mathrm{P}$ and NP rats (Fig. S1).

To examine whether $3 \alpha, 5 \alpha$-THP inhibits TLR7 signal activation we studied female $\mathrm{P}$ rats that were treated (30 min) with $3 \alpha, 5 \alpha-$ THP $(15 \mathrm{mg} / \mathrm{kg})$ or vehicle control (45\% w/v 2-hydroxypropyl- $\beta$-cyclodextrin). We found that in the NAc from female P rats, pIRF7 expression was inhibited by $3 \alpha, 5 \alpha$-THP by $34.1 \pm 11.1 \%$ ( $t$-test: $t=2.417$, $\mathrm{df}=14, p=0.0299, n=8 /$ grp ) (Fig. 2d) but the levels of TLR7 were not altered by $3 \alpha, 5 \alpha$-THP ( $t$-test: $t=0.7286$, $\mathrm{df}=14, p=0.4782, n=8 /$ grp ) (Fig. 2e) indicating that $3 \alpha, 5 \alpha-$ THP inhibits TLR7 signal activation but not protein expression. $3 \alpha, 5 \alpha$-THP also inhibited pIRF7 expression in the female $\mathrm{P}$ rat amygdala by $41.5 \pm 8.0 \%$ ( $t$-test: $t=2.423, \mathrm{df}=14, p=0.0296, n=8 /$ grp). In contrast, we found no indication of innate activation of TLR2 and TLR3 signals in P rat brain (Fig. S2A, B) and $3 \alpha, 5 \alpha-$ THP had no effect on pIRF3 in P rat NAc (Fig. S2C). The data support the conclusion that $3 \alpha, 5 \alpha$-THP inhibits the MyD88-dependent TLR signals, also in the brain.

\section{3a,5a-THP inhibits MyD88, but not TRIF binding to TLRs}

To further confirm that $3 \alpha, 5 \alpha$-THP inhibits MyD88but not TRIF- dependent signals and examine the potential mechanism of inhibition, protein extracts from the NAc of male and female P rats treated with $3 \alpha, 5 \alpha-$ THP or vehicle control ( $n=3-4 /$ grp $)$ were immunoprecipitated with antibody to MyD88 or TRIF. The MyD88 and TRIF precipitates from both males and females were immunoblotted with antibody to TLR4 and the MyD88 precipitates from females were immunoblotted with antibody to TLR7. Immunoblotting with MyD88 or TRIF antibodies served to confirm immunoprecipitation and normal IgG was used as immunoprecipitating antibody control. We found that MyD88 co-precipitates with TLR4 and TLR7 and TRIF co-precipitates with TLR4. The levels of TLR4 co-precipitating with MyD88 were significantly reduced by $3 \alpha, 5 \alpha-$ THP in both males $(65.9 \pm$ $15.9 \%$ by $t$-test: $t=3.237, \mathrm{df}=4, p=0.0318, n=3 /$ grp) and females $(62.3 \pm 12.4 \%$ by $t$-test: $t=2.596, \mathrm{df}=6$, $p=0.0409, n=4 /$ grp). $3 \alpha, 5 \alpha-$ THP also inhibited the levels of TLR7 co-precipitating with MyD88 in females 


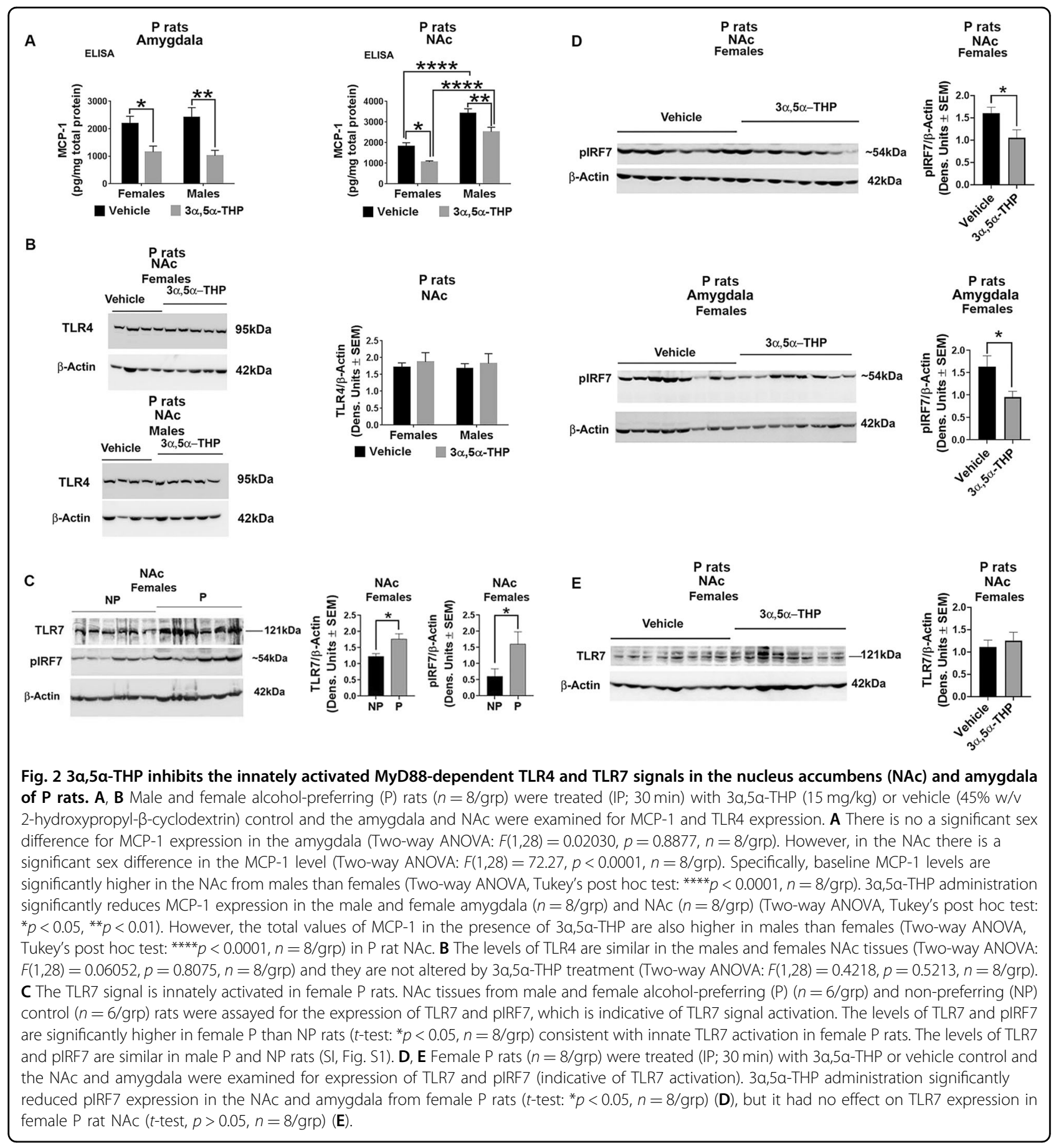

$(57.7 \pm 8.8 \%$ by $t$-test: $t=3.500, \mathrm{df}=4, p=0.0249, n=3$ / grp) (Fig. 3a, b), but it did not alter the levels of TLR4/ TRIF binding both in males ( $t$-test: $t=0.5593, \mathrm{df}=4$, $p=0.6058, n=3 /$ grp $)$ or females ( $t$-test: $t=0.5682$, $\mathrm{df}=4, p=0.6003, n=3 /$ grp) (Fig. 3c). Collectively, the data indicate that $3 \alpha, 5 \alpha$-THP inhibits MyD88, but not TRIF binding to TLRs, thereby inhibiting the MyD88but not the TRIF-dependent signal.

\section{Discussion}

The salient feature of the data presented in this report is the finding that $3 \alpha, 5 \alpha-$ THP inhibits the activation of MyD88- but not TRIF-dependent TLR signals in cultured macrophage cells and brain, and that brain site and sex appear to affect the resulting pro-inflammatory response. Since most TLRs signal through MyD88-dependent pathways, it would appear that the endogenous neurosteroid 


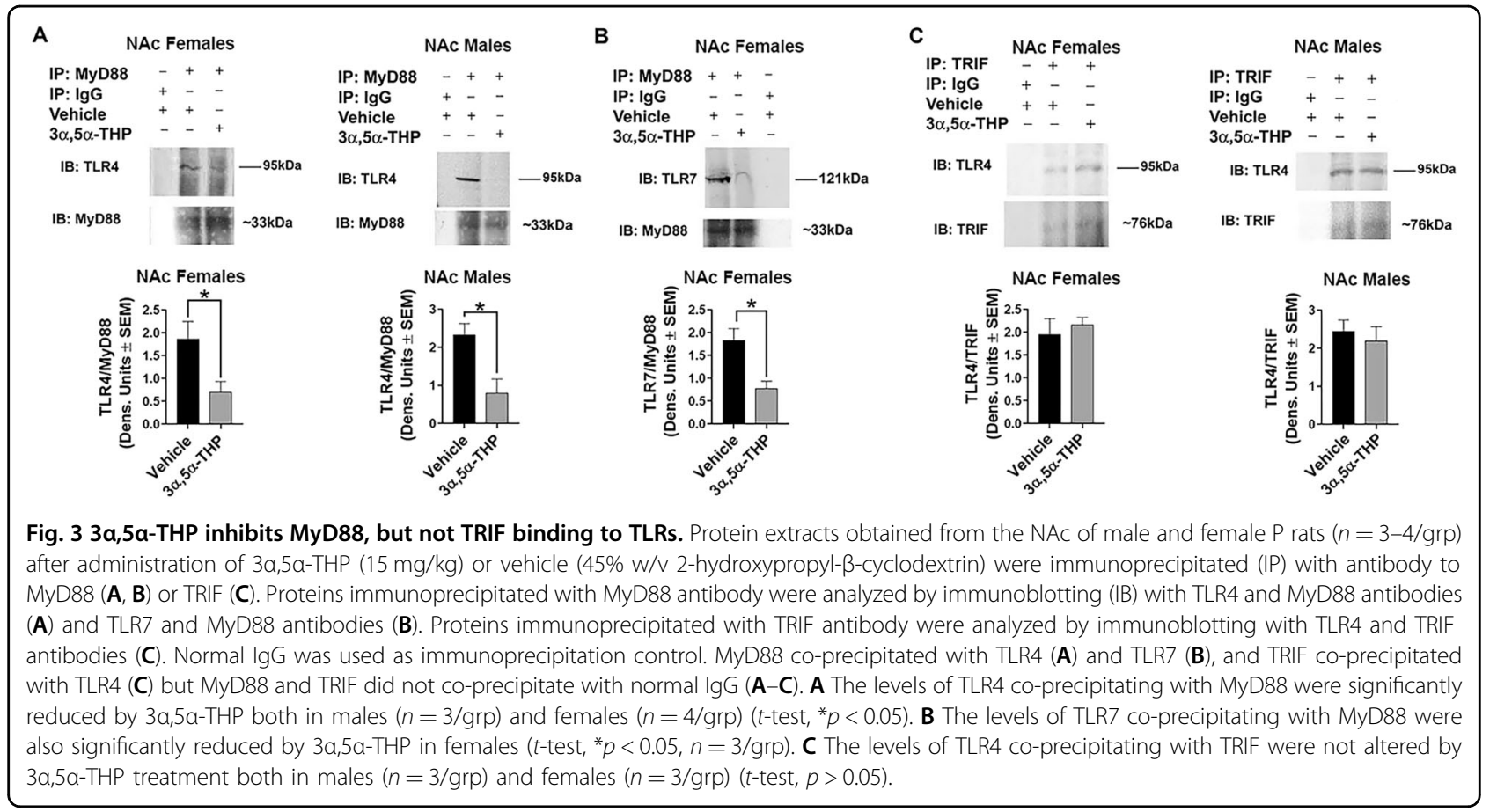

$3 \alpha, 5 \alpha$-THP has remarkable and heretofore unappreciated potential to prevent or ameliorate inflammatory pathway signals and possibly inflammatory conditions.

TLR signals impact infectious diseases and noninfectious CNS disorders, learning, memory, and neurogenesis $^{18,61,62}$. The TLR4 signal contributes to various neuropsychiatric conditions, addiction ${ }^{3,9,63}$, and depression $^{8}$. The TLR2 signal is involved in Alzheimer's disease pathogenesis ${ }^{64}$ and multiple sclerosis brain lesions ${ }^{65}$. The TLR7 signal enhances contextual fear memory and depression-like behaviors in mice ${ }^{66}$ and the TLR3 signal is involved in anxiety-related behaviors, impairs motor coordination, and cues fear memory ${ }^{12}$. Both TLR4 and TLR3 are associated with alcohol consumption, albeit in distinct models and at different stages. The innately activated TLR4 signal in $\mathrm{P}$ rats regulates the voluntary initiation of alcohol consumption ${ }^{9,34,35,59}$ while Poly(I:C)induced TLR3/TRIF-dependent signaling was seen in C57BL/6J mice maintained on an alcohol diet ${ }^{67}$.

TLRs share common structural domains, which define their ability to recruit the adaptor proteins that regulate signaling. All TLRs except for TLR3, signal through the MyD88 adaptor, the recruitment of which requires binding of the bridging adaptor MyD88 adaptor-like (MAL)/ TIR domain-containing adaptor protein (TIRAP) ${ }^{2,68}$. TLR3 signals exclusively via the TRIF adaptor, and TLR4 signals through both MyD88 and TRIF ${ }^{2}$. TRIF recruitment requires binding of the bridging adaptor TRIF-related adaptor molecule ${ }^{2,69}$. Available data suggest that the TLR4/MyD88 pathway positively regulates the expression of pro-inflammatory cytokines (viz. TNF- $\alpha$, IL-6, IL-1) and chemokines (viz. MCP-1) ( $^{6,35,70,71}$ and microglial pro-inflammatory (M1) polarization ${ }^{72}$, but the TLR4/TRIF signal may be anti-inflammatory and neuroprotective $^{73-76}$.

Since $3 \alpha, 5 \alpha$-THP inhibits the activated TLR4 signaling pathway in cultured cells, but it has no effect on the steady state non-activated pathway ${ }^{31}$, we first studied its effects on other TLRs in RAW246.7 cells using ligands specific for the activation of TLRs located on the plasma membrane (TLR2) or endosomes (TLR7, TLR3) that signal exclusively through MyD88 (TLR2, TLR7) or TRIF (TLR3) binding ${ }^{2,48-52}$. The ligands were used at the lowest dose that induced maximal activation responses as determined in independent studies and previously confirmed by us ${ }^{31,56-58}$. We found that $3 \alpha, 5 \alpha-\mathrm{THP}(1.0 \mu \mathrm{M})$ inhibits the activation of both cell surface and endosomal TLR signals that are MyD88-dependent, but it has no effect on the TRIF-dependent signal. Our previous data indicate that TLR inhibition levels are similar for $3 \alpha, 5 \alpha-$ $\mathrm{THP}$ at 0.5 and $1.0 \mu \mathrm{M}^{31}$. However, additional studies are needed in order to define the potency of $3 \alpha, 5 \alpha$-THP in inhibiting all of the TLR pathways.

We found no indication of innate activation of TLR3 signals both in male and female P rat brain and TLR7 signal is activated in female but not male $P$ rat brain as indicated by higher levels of TLR7 and PIRF7 in P vs. NP rat brains. TLR7 is encoded by an X chromosome locus and in female somatic cells there is a random silencing of one of the two X chromosomes ${ }^{77,78}$. However, 
for example, TLR7 may escape the X chromosome inactivation in immune cells ${ }^{77}$. The increased levels of TLR7 in female $\mathrm{P}$ vs. NP rat brains may be due to the reactivation of the second $\mathrm{X}$ chromosome controlled by noncoding RNAs, such as $\mathrm{Xist}^{79}$. 3 $\alpha, 5 \alpha$-THP inhibited expression of the activated TLR7 signal pIRF7 in female $\mathrm{P}$ rat brain. Specifically, the reduction of pIRF7 was similar $(\sim 35-40 \%)$ in the NAc and amygdala from female $P$ rats, indicating this effect is not isolated to NAc. Significantly, $3 \alpha, 5 \alpha$-THP did not inhibit pIRF3 expression (TRIF-dependent TLR3 and TLR4 signal) and coimmunoprecipitation studies confirmed that $3 \alpha, 5 \alpha$-THP inhibits MyD88 binding to TLR4 and TLR7, but not TRIF binding to TLR3 or TLR4 in P rat NAc lysates. Collectively, the data indicate that $3 \alpha, 5 \alpha$-THP inhibits MyD88, but not TRIF-dependent TLR signaling (Fig. 4).

The identity of the innate agonists that activate the TLR signals in the brain is unclear. We have shown that in neurons, the TLR4 signal is activated by binding with the $\alpha 2$ subunit of $\mathrm{GABA}_{\mathrm{A}}$ receptors ${ }^{9,34,59}$, but the role of the $\alpha 2$ subunit in the activation of other TLR signals and in other cell types is still unclear. The differential TLR4 and TLR7 signal activation in a sex-dependent manner and/or the potential TLR-TLR cross talk and its resulting signal regulatory outcome are also not well understood. For example, the neuropeptide corticotropin-releasing factor (CRF) alters different circuits in males and females with brain region specific sensitivity ${ }^{80}$ and it promotes sex differences in the reinforcing effects of nicotine ${ }^{81}$. CRF regulates an amplification loop for the innately

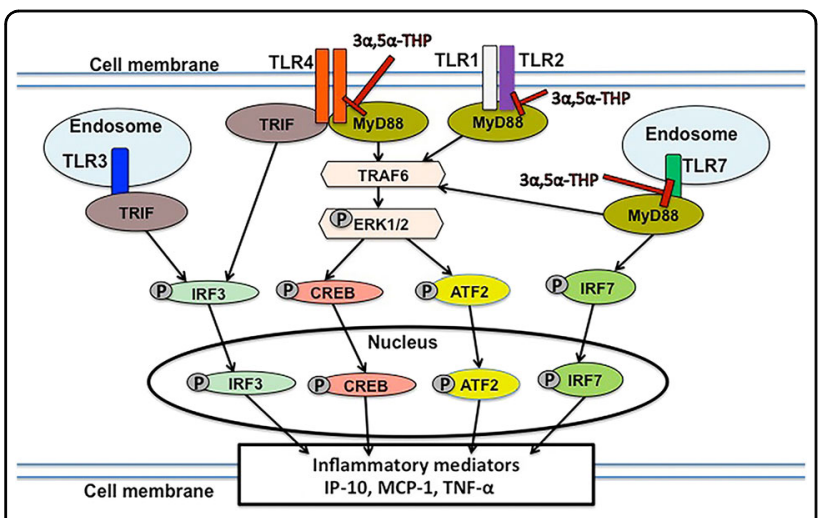

Fig. 4 Schematic of TLR signal inhibition by $\mathbf{3 a}, \mathbf{5 a}$-THP. TLR4, TLR2, and TLR7 signal activation initiates with TLR binding of MyD88, and this binding is inhibited by $3 a, 5 a-T H P$. This results in the inhibition of pathway activation, including TRAF6 and activated (phosphorylated) downstream members (pERK1/2, pCREB, pATF2, and pIRF7). The activated transcription factors translocate to the nucleus and initiate the production of various inflammatory mediators (IP-10, MCP-1, and TNF-a). TLR4 also binds TRIF, which is the only adaptor protein bound by TLR3, leading to the phosphorylation (activation) of IRF3.

Significantly, 3a,5a-THP does not inhibit TRIF binding and its resulting signal activation. activated TLR4 signal that is focused on pERK1/2, downstream of TRAF6 activation $^{32}$, a site that also applies to TLR7 signaling (Fig. 4). Also, the endogenous TLR4 agonist HMGB1 facilitates the release of the TLR7 agonist miRNA let- $7 \mathrm{~b}^{82}$ indicative of TLR4/TLR7 cross talk. This cross talk is likely not involved in signal activation in our study, because $3 \alpha, 5 \alpha$-THP inhibits LPSinduced HMGB1 increase ${ }^{31}$ and we find that in the $\mathrm{P}$ rat NAc, the TLR4 signal is primarily activated in males and the TLR7 signal is only activated in females, but not in males. Increased TLR4 activation in males as opposed to TLR7 in females may also reflect antagonistic effects of TLRs cross talk, as suggested by the finding that the TLR7 ligand miR-142-3p inhibits TLR4 activation and TLR4 represses miR-142-3p expression ${ }^{83-85}$. However, the possibility of TLR4/TLR7 cross talk in macrophages and at brain sites without sex differences cannot be excluded. Also MCP-1 is expressed in neurons, astrocytes, and microglia ${ }^{35,86-88}$ and it is still unclear whether the $3 \alpha, 5 \alpha$-THP-mediated inhibition is cell type specific. Moreover, MyD88 regulates signaling downstream of interleukin-1 receptor (IL-1R) ${ }^{89,90}$, but the effect of $3 \alpha, 5 \alpha-$ THP on IL-1R/MyD88 signaling is unknown. Ongoing studies are designed to address these questions.

Our data indicate that $3 \alpha, 5 \alpha$-THP inhibits TLR binding

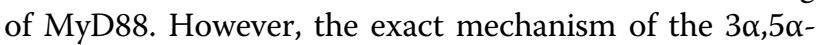
THP inhibitory effect is also still unclear. One possibility is that $3 \alpha, 5 \alpha$-THP binds MyD88 or the bridging adaptor protein MAL/TIRAP thereby preventing MyD88 recruitment to the TLRs. $3 \alpha, 5 \alpha$-THP could also cause the degradation of bound MyD88, as was recently shown for pregnenolone that promotes the ubiquitination and degradation of TIRAP and TLR2 in human embryonic HEK293T cells $^{29}$. Further studies are needed in order to detect the proteins that are bound by $3 \alpha, 5 \alpha$-THP, determine their localization with single-molecule resolution, and enable quantification and kinetic analysis. This is particularly relevant since inhibition of protein binding may require specific ring structures as suggested by the finding that the structure of the steroid D ring common to $3 \alpha, 5 \alpha-$ THP, progesterone and pregnenolone, is associated with inhibition of pro-inflammatory signals ${ }^{19-21,31,91}$. The potential cross talk between diverse activated TLR pathways, their ability to potentially compensate for $3 \alpha, 5 \alpha-$ THP-inhibited signals ${ }^{92}$ and differentially affect other cell types (viz. neurons-microglia) are additional questions in need of further elucidation.

The IP administration of $3 \alpha, 5 \alpha$-THP (30 min) inhibited the expression of MCP-1 (MyD88-dependent TLR signal) both in male and female $\mathrm{P}$ rat brain. Specifically, in the amygdala the reduction of MCP-1 was similar ( $50-55 \%)$ in males and females, however in the NAc, inhibition was only $\sim 25 \%$ in males compared to $\sim 40 \%$ in females, while the total levels of MCP-1 in the NAc are significantly 
higher in males than females, both in the vehicle- and $3 \alpha, 5 \alpha$-THP-treated P rats. Sexually dimorphic behaviors in $P$ rats are unknown and the significance of sex differences in TLR4 and TLR7 activation in P rat brain is unclear. However, they may be related to sex differences in ethanol consumption ( $\mathrm{F} \gg \mathrm{M})$ as shown for Sprague-Dawley rats ${ }^{93}$. Future studies will use state-ofthe-art mass spectroscopic methods to determine the serum or brain levels of allopregnanolone reached after treatment with allopregnanolone vs. vehicle in both males and females in order to determine whether the impact of allopregnanolone on the regulation of inflammation occurs at supra-physiological or physiological levels that may be present at rest, after stress, or in estrous phases with higher allopregnanolone levels in females.

In summary, the ability of $3 \alpha, 5 \alpha$-THP to inhibit MyD88dependent TLR signals suggests a wide applicability to many inflammatory conditions that involve TLR activation of pro-inflammatory pathways ${ }^{27,28,31}$. Since neurosteroids, like immune factors, circulate in the bloodstream, cross the blood brain barrier, diffuse between different cell types, and exhibit paracrine effects in many cells, it is likely that neurosteroids are endogenous modulators of TLR activation and may contribute to inflammatory disease susceptibility and recovery. These results have applicability to many conditions involving pro-inflammatory TLR activation of cytokines, chemokines, and interferons.

\section{Acknowledgements}

This work was supported by NIH grant R01-AA024095, Sage Therapeutics, and the Bowles Center for Alcohol Studies at UNC School of Medicine to ALM. We thank Dr. Richard L. Bell for providing alcohol-preferring $P$ rats for these studies through NIAAA Resource funding U24AA015512.

\section{Conflict of interest}

The authors A.L.M., L.A., and I.B. hold a provisional US patent for the mechanisms of 3a,5a-THP action reported in this manuscript. The authors declare no other conflicts of interest.

\section{Publisher's note}

Springer Nature remains neutral with regard to jurisdictional claims in published maps and institutional affiliations.

Supplementary information The online version contains supplementary material available at https://doi.org/10.1038/s41398-021-01266-1.

Received: 10 June 2020 Revised: 21 January 2021 Accepted: 2 February 2021

Published online: 26 February 2021

\section{References}

1. Kawai, T. \& Akira, S. TLR signaling. Semin. Immunol. 19, 24-32 (2007)

2. Gay, N. J., Symmons, M. F., Gangloff, M. \& Bryant, C. E. Assembly and localization of Toll-like receptor signalling complexes. Nat. Rev. Immunol. 14, 546-558 (2014).

3. Lehnardt, S. et al. Activation of innate immunity in the CNS triggers neurodegeneration through a toll-like receptor 4-dependent pathway. Proc. Natl Acad. Sci. USA 100, 8514-8519 (2003).
4. Flajnik, M. F. A cold-blooded view of adaptive immunity. Nat. Rev. Immunol. 18, 438-453 (2018).

5. Shmueli, A., Shalit, T., Okun, E. \& Shohat-Ophir, G. The toll pathway in the central nervous system of flies and mammals. Neuromolecular Med. 20, 419-436 (2018).

6. Kawasaki, T. \& Kawai, T. Toll-like receptor signaling pathways. Front. Immunol. 5, 461 (2014).

7. Bullmore, E. The art of medicine: inflamed depression. Lancet 392, 1189-1190 (2018).

8. Liu, J., Buisman-Pijlman, F. \& Hutchinson, M. R. Toll-like receptor 4: innate immune regulator of neuroimmune and neuroendocrine interactions in stress and major depressive disorder. Front. Neurosci. 8, 309 (2014).

9. Balan, I. et al. The GABAA receptor alpha2 subunit activates a neuronal TLR4 signal in the ventral tegmental area that regulates alcohol and nicotine abuse. Brain Sci 8, 72 (2018).

10. Crews, F. T., Lawrimore, C. J., Walter, T. J. \& Coleman, L. G. Jr The role of neuroimmune signaling in alcoholism. Neuropharmacology 122, 56-73 (2017).

11. Shi, H., Hua, X., Kong, D., Stein, D. \& Hua, F. Role of Toll-like receptor mediated signaling in traumatic brain injury. Neuropharmacology 145, 259-267 (2019).

12. Okun, E. et al. Toll-like receptor 3 inhibits memory retention and constrains adult hippocampal neurogenesis. Proc. Natl Acad. Sci. USA 107, 15625-15630 (2010).

13. Okun, E. et al. Toll-like receptors in neurodegeneration. Brain Res. Rev. $\mathbf{5 9}$ 278-292 (2009).

14. Gesuete, R., Kohama, S. G. \& Stenzel-Poore, M. P. Toll-like receptors and ischemic brain injury. J. Neuropathol. Exp. Neurol. 73, 378-386 (2014).

15. Vezzani, A., French, J., Bartfai, T. \& Baram, T. Z. The role of inflammation in epilepsy. Nat. Rev. Neurol. 7, 31-40 (2011).

16. Maroso, M. et al. Interleukin-1 type 1 receptor/Toll-like receptor signalling in epilepsy: the importance of IL-1 beta and high-mobility group box 1. J. Intern. Med. 270, 319-326 (2011).

17. Wittebole, X., Castanares-Zapatero, D. \& Laterre, P. F. Toll-like receptor 4 modulation as a strategy to treat sepsis. Mediators Inflamm. 2010, 568396 (2010).

18. Del Valle, D. M. et al. An inflammatory cytokine signature predicts COVID-19 severity and survival. Nat. Med. 26, 1636-1643 (2020).

19. Stell, B. M., Brickley, S. G., Tang, C. Y., Farrant, M. \& Mody, I. Neuroactive steroids reduce neuronal excitability by selectively enhancing tonic inhibition mediated by delta subunit-containing GABAA receptors. Proc. Natl Acad. Sci. USA 100, 14439-14444 (2003).

20. Majewska, M. D., Harrison, N. L., Schwartz, R. D., Barker, J. L. \& Paul, S. M. Steroid hormone metabolites are barbiturate-like modulators of the GABA receptor. Science 232, 1004-1007 (1986).

21. Paul, S. M. \& Purdy, R. H. Neuroactive steroids. FASEB J. 6, 2311-2322 (1992).

22. Patchev, V. K., Shoaib, M., Holsboer, F. \& Almeida, O. F. The neurosteroid tetrahydroprogesterone counteracts corticotropin-releasing hormoneinduced anxiety and alters the release and gene expression of corticotropinreleasing hormone in the rat hypothalamus. Neuroscience 62, 265-271 (1994).

23. Wright, D. W. et al. ProTECT: a randomized clinical trial of progesterone for acute traumatic brain injury. Ann. Emerg. Med. 49, 391-402, e1-2 (2007).

24. Marx, C. E. et al. Pregnenolone as a novel therapeutic candidate in schizophrenia: emerging preclinical and clinical evidence. Neuroscience 191, 78-90 (2011).

25. Milivojevic, V., Fox, H. C., Sofuoglu, M., Covault, J. \& Sinha, R. Effects of progesterone stimulated allopregnanolone on craving and stress response in cocaine dependent men and women. Psychoneuroendocrinology 65, 44-53 (2016).

26. Meltzer-Brody, S. et al. Brexanolone injection in post-partum depression: two multicentre, double-blind, randomised, placebo-controlled, phase 3 trials. Lancet 392, 1058-1070 (2018).

27. Boero, G., Porcu, P. \& Morrow, A. L. Pleiotropic actions of allopregnanolone underlie therapeutic benefits in stress-related disease. Neurobiol. Stress $\mathbf{1 2}$, 100203 (2020).

28. Morrow, A. L, Boero, G. \& Porcu, P. A rationale for allopregnanolone treatment of alcohol use disorders: basic and clinical studies. Alcohol Clin. Exp. Res. $\mathbf{4 4}$ 320-339 (2020).

29. Murugan, S., Jakka, P., Namani, S., Mujumdar, V. \& Radhakrishnan, G. The neurosteroid pregnenolone promotes degradation of key proteins in the innate immune signaling to suppress inflammation. J. Biol. Chem. 294, 4596-4607 (2019). 
30. He, J., Evans, C. O., Hoffman, S. W., Oyesiku, N. M. \& Stein, D. G. Progesterone and allopregnanolone reduce inflammatory cytokines after traumatic brain injury. Exp. Neurol. 189, 404-412 (2004).

31. Balan, I., Beattie, M. C., O'Buckley, T. K., Aurelian, L. \& Morrow, A. L. Endogenous neurosteroid (3alpha,5alpha)3-hydroxypregnan-20-one inhibits toll-like-4 receptor activation and pro-inflammatory signaling in macrophages and brain. Sci. Rep. 9, 1220 (2019)

32. Balan, I., Warnock, K. T., Puche, A., Gondre-Lewis, M. C. \& Aurelian, L. Innately activated TLR4 signal in the nucleus accumbens is sustained by CRF amplification loop and regulates impulsivity. Brain Behav. Immun. 69, 139-153 (2018).

33. Aurelian, L., Warnock, K. T., Balan, I., Puche, A. \& June, H. TLR4 signaling in VTA dopaminergic neurons regulates impulsivity through tyrosine hydroxylase modulation. Transl. Psychiatry 6, e815 (2016).

34. Liu, J. et al. Binge alcohol drinking is associated with GABAA alpha2-regulated Toll-like receptor 4 (TLR4) expression in the central amygdala. Proc. Natl Acad. Sci. USA 108, 4465-4470 (2011).

35. June, H. L. et al. CRF-amplified neuronal TLR4/MCP-1 signaling regulates alcohol self-administration. Neuropsychopharmacology 40, 1549-1559 (2015).

36. McBride, W. J. et al. Gene expression in the ventral tegmental area of 5 pairs of rat lines selectively bred for high or low ethanol consumption. Pharm. Biochem. Behav. 102, 275-285 (2012).

37. Knapp, D. J. et al. Effects of a stressor and corticotrophin releasing factor on ethanol deprivation-induced ethanol intake and anxiety-like behavior in alcohol-preferring P rats. Psychopharmacology 218, 179-189 (2011).

38. Stewart, R. B., Gatto, G. J., Lumeng, L., Li, T. K. \& Murphy, J. M. Comparison of alcohol-preferring (P) and nonpreferring (NP) rats on tests of anxiety and for the anxiolytic effects of ethanol. Alcohol 10, 1-10 (1993).

39. McKinzie, D. L. et al. Acoustic startle and fear-potentiated startle in alcoholpreferring (P) and -nonpreferring (NP) lines of rats. Pharm. Biochem. Behav. 65 691-696 (2000).

40. Ogle, T. F. \& Kitay, J. I. Ovarian and adrenal steroids during pregnancy and the oestrous cycle in the rat. J. Endocrinol. 74, 89-98 (1977).

41. Sze, Y. \& Brunton, P. J. Sex, stress and steroids. Eur. J. Neurosci. 52, 2487-2515 (2020).

42. Crawley, J. N., Glowa, J. R., Majewska, M. D. \& Paul, S. M. Anxiolytic activity of an endogenous adrenal steroid. Brain Res. 398, 382-385 (1986).

43. Devaud, L. L., Purdy, R. H. \& Morrow, A. L. The neurosteroid, 3 alpha-hydroxy-5 alpha-pregnan-20-one, protects against bicuculline-induced seizures during ethanol withdrawal in rats. Alcohol Clin. Exp. Res. 19, 350-355 (1995)

44. Mendelson, W. B. et al. Sleep induction by an adrenal steroid in the rat. Psychopharmacology 93, 226-229 (1987).

45. Balan I., Beattie M. C., O'Buckley T. K., Aurelian L. \& Morrow A. L. Protective effects of the endogenous neurosteroid (3a,5a)3-hydroxypregnan-20-one (3a,5a-THP) on proinflammatory TLR4 signaling in immune cells and brain. Soc. Neuroscience Meeting Planner, Online Abstract 659.05 (2018).

46. Berghaus, L. J. et al. Innate immune responses of primary murine macrophage-lineage cells and RAW 264.7 cells to ligands of Toll-like receptors 2, 3, and 4. Comp. Immunol. Microbiol. Infect. Dis. 33, 443-454 (2010).

47. Li, J.-Y., Liu, Y., Gao, X.-X., Gao, X. \& Cai, H. TLR2 and TLR4 signaling pathways are required for recombinant Brucella abortus BCSP31-induced cytokine production, functional upregulation of mouse macrophages, and the Th1 immune response in vivo and in vitro. Cell. Mol. Immunol. 11, 477-494 (2014).

48. Nie, $X$. et al. The innate immune receptors TLR2/4 mediate repeated social defeat stress-induced social avoidance through prefrontal microglial activation. Neuron 99, 464-479 e7 (2018).

49. Amiresmaeili, A., Roohollahi, S., Mostafavi, A. \& Askari, N. Effects of oregano essential oil on brain TLR4 and TLR2 gene expression and depressive-like behavior in a rat model. Res. Pharm. Sci. 13, 130-141 (2018).

50. Hemmi, H. et al. Small anti-viral compounds activate immune cells via the TLR7 MyD88-dependent signaling pathway. Nat. Immunol. 3, 196-200 (2002).

51. Yamamoto, M. et al. Role of adaptor TRIF in the MyD88-independent toll-like receptor signaling pathway. Science 301, 640-643 (2003).

52. Leonard, J. N. et al. The TLR3 signaling complex forms by cooperative receptor dimerization. Proc. Natl Acad. Sci. USA 105, 258-263 (2008).

53. Moynagh, P. N. TLR signalling and activation of IRFs: revisiting old friends from the NF-kappaB pathway. Trends Immunol. 26, 469-476 (2005).

54. Park, C. et al. TLR3-mediated signal induces proinflammatory cytokine and chemokine gene expression in astrocytes: differential signaling mechanisms of TLR3-induced IP-10 and IL-8 gene expression. Glia 53, 248-256 (2006).
55. Zhu, J. et al. High-throughput screening for TLR3-IFN regulatory factor 3 signaling pathway modulators identifies several antipsychotic drugs as TLR inhibitors. J. Immunol. 184, 5768-5776 (2010).

56. Ge, Y. et al. Rotavirus NSP4 triggers secretion of proinflammatory cytokines from macrophages via toll-like receptor 2. J. Virol. 87, 11160-11167 (2013).

57. Wang, J. et al. Effect of TLR agonists on the differentiation and function of human monocytic myeloid-derived suppressor cells. J. Immunol. 194, 4215-4221 (2015).

58. Guinn, Z., Lampe, A. T., Brown, D. M. \& Petro, T. M. Significant role for IRF3 in both $\mathrm{T}$ cell and APC effector functions during $\mathrm{T}$ cell responses. Cell Immunol. 310, 141-149 (2016).

59. Aurelian, L. \& Balan, I. GABAAR alpha2-activated neuroimmune signal controls binge drinking and impulsivity through regulation of the CCL2/CX3CL1 balance. Psychopharmacology 236, 3023-3043 (2019).

60. Liberman, A. C. et al. Neuroimmune and inflammatory signals in complex disorders of the central nervous system. Neuroimmunomodulation $\mathbf{2 5}$, 246-270 (2018).

61. Okun, E., Griffioen, K. J. \& Mattson, M. P. Toll-like receptor signaling in neural plasticity and disease. Trends Neurosci. 34, 269-281 (2011).

62. Sawa, A. \& Roger, T. Targeting toll-like receptors: promising therapeutic strategies for the management of sepsis-associated pathology and infectious diseases. Front. Immunol. 4, 387 (2013).

63. Crews, F. T., Qin, L., Sheedy, D., Vetreno, R. P. \& Zou, J. High mobility group box $1 /$ Toll-like receptor danger signaling increases brain neuroimmune activation in alcohol dependence. Biol. Psychiatry 73, 602-612 (2013).

64. Jana, M., Palencia, C. A. \& Pahan, K. Fibrillar amyloid-beta peptides activate microglia via TLR2: implications for Alzheimer's disease. J. Immunol. 181, 7254-7262 (2008).

65. Sloane, J. A. et al. Hyaluronan blocks oligodendrocyte progenitor maturation and remyelination through TLR2. Proc. Natl Acad. Sci. USA 107, 11555-11560 (2010).

66. Hung, Y. F., Chen, C. Y., Li, W. C., Wang, T. F. \& Hsueh, Y. P. Tlr7 deletion alters expression profiles of genes related to neural function and regulates mouse behaviors and contextual memory. Brain Behav. Immun. 72, 101-113 (2018).

67. Warden, A. S. et al. Toll-like receptor 3 activation increases voluntary alcohol intake in C57BL/6J male mice. Brain Behav. Immun. 77, 55-65 (2019).

68. Fitzgerald, K. A. et al. Mal (MyD88-adapter-like) is required for toll-like receptor4 signal transduction. Nature 413, 78-83 (2001).

69. Tanimura, N., Saitoh, S., Matsumoto, F., Akashi-Takamura, S. \& Miyake, K. Roles for LPS-dependent interaction and relocation of TLR4 and TRAM in TRIFsignaling. Biochem. Biophys. Res. Commun. 368, 94-99 (2008).

70. Akhter, N. et al. TLR4/MyD88 -mediated CCL2 production by lipopolysaccharide (endotoxin): Implications for metabolic inflammation. J. Diabetes Metab. Disord. 17, 77-84 (2018).

71. Grassin-Delyle, S. et al. The role of toll-like receptors in the production of cytokines by human lung macrophages. J. Innate Immun. 12, 63-73 (2020).

72. Gong, L. et al. Toll-Interleukin 1 receptor domain-containing adaptor protein positively regulates BV2 cell M1 polarization. Eur. J. Neurosci. 43, 1674-1682 (2016).

73. Chung, $H$. J. et al. The anti-inflammatory effects of Shinbaro3 is mediated by downregulation of the TLR4 signalling pathway in IPS-stimulated RAW 264.7 macrophages. Mediators Inflamm. 2018, 4514329 (2018).

74. Vartanian, K. B. et al. LPS preconditioning redirects TLR signaling following stroke: TRIF-IRF3 plays a seminal role in mediating tolerance to ischemic injury. J. Neuroinflammation 8, 140 (2011).

75. Chen, S. et al. Valproic acid attenuates traumatic spinal cord injury-induced inflammation via STAT1 and NF-kappaB pathway dependent of HDAC3. J. Neuroinflammation 15, 150 (2018).

76. Marsh, B. et al. Systemic lipopolysaccharide protects the brain from ischemic injury by reprogramming the response of the brain to stroke: a critical role for IRF3. J. Neurosci. 29, 9839-9849 (2009).

77. Souyris M. et al. TLR7 escapes X chromosome inactivation in immune cells. Sci. Immunol. 3, eaap8855 (2018)

78. Butchi, N. B. Pourciau, S., Du, M. Morgan, T. W. \& Peterson, K. E. Analysis of the neuroinflammatory response to TLR7 stimulation in the brain: comparison of multiple TLR7 and/or TLR8 agonists. J. Immunol. 180, 7604-7612 (2008).

79. Adrianse, R. L. et al. Perturbed maintenance of transcriptional repression on the inactive $X$-chromosome in the mouse brain after Xist deletion. Epigenetics Chromatin 11, 50 (2018). 
80. Salvatore, M. et al. Sex differences in circuits activated by corticotropin releasing factor in rats. Horm. Behav. 97, 145-153 (2018).

81. Uribe, K. P. et al. Overexpression of corticotropin-releasing factor in the nucleus accumbens enhances the reinforcing effects of nicotine in intact female versus male and ovariectomized female rats. Neuropsychopharmacology $\mathbf{4 5}$, 394-403 (2020)

82. Coleman, L. G. Jr., Zou, J. \& Crews, F. T. Microglial-derived miRNA let-7 and HMGB1 contribute to ethanol-induced neurotoxicity via TLR7. J. Neuroinflammation 14, 22 (2017).

83. Dombkowski, A. A. et al. TLR7 activation in epilepsy of tuberous sclerosis complex. Inflamm. Res. 68, 993-998 (2019).

84. Fordham, J. B., Naqvi, A. R. \& Nares, S. Regulation of miR-24, miR-30b, and miR142-3p during macrophage and dendritic cell differentiation potentiates innate immunity. J. Leukoc. Biol. 98, 195-207 (2015).

85. Sun, Y. et al. PU.1-dependent transcriptional regulation of miR-142 contributes to its hematopoietic cell-specific expression and modulation of IL-6. J. Immunol. 190, 4005-4013 (2013).

86. Harper, K. M. et al. Phenotyping CCL2 containing central amygdala neurons controlling alcohol withdrawal-induced anxiety. Front. Cell. Neurosci. 14, 296 (2020).
87. Banisadr, G. et al. Highly regionalized neuronal expression of monocyte chemoattractant protein-1 (MCP-1/CCL2) in rat brain: evidence for its colocalization with neurotransmitters and neuropeptides. J. Comp. Neurol. 489, 275-292 (2005).

88. Yao, Y. \& Tsirka, S. E. Monocyte chemoattractant protein-1 and the blood-brain barrier. Cell Mol. Life Sci. 71, 683-697 (2014).

89. Davis, C. N. et al. MyD88-dependent and -independent signaling by IL-1 in neurons probed by bifunctional Toll/IL-1 receptor domain/BB-loop mimetics. Proc. Natl Acad. Sci. USA 103, 2953-2958 (2006).

90. Deguine, J. \& Barton, G. M. MyD88: a central player in innate immune signaling. F1000Prime Rep. 6, 97 (2014).

91. Harrison, N. L., Majewska, M. D., Harrington, J. W. \& Barker, J. L. Structure-activity relationships for steroid interaction with the gamma-aminobutyric acidA receptor complex. J. Pharm. Exp. Ther. 241, 346-353 (1987).

92. Kim, J. S., Kim, Y. R. \& Yang, C. S. Latest comprehensive knowledge of the crosstalk between TLR signaling and mycobacteria and the antigens driving the process. J. Microbiol. Biotechnol. 29, 1506-1521 (2019).

93. Li, J. et al. Differences between male and female rats in alcohol drinking, negative affects and neuronal activity after acute and prolonged abstinence. Int J. Physiol. Pathophysiol. Pharm. 11, 163-176 (2019). 